\title{
Immunology and Immunodiagnosis of Cystic Echinococcosis: An Update
}

\author{
Wenbao Zhang, ${ }^{1,2}$ Hao Wen, ${ }^{1}$ Jun Li, ${ }^{1,2}$ Renyong Lin, ${ }^{1}$ and Donald P. McManus ${ }^{2}$ \\ ${ }^{1}$ Clinical Medical Research Institute, First Affiliated Hospital of Xinjiang Medical University, No. 1 Liyushan Road, Urumqi, \\ Xinjiang 830054, China \\ ${ }^{2}$ Molecular Parasitology Laboratory, Infectious Diseases Division, Queensland Institute of Medical Research, Brisbane, \\ QLD 4029, Australia
}

Correspondence should be addressed to Wenbao Zhang, wenbaozhang62@yahoo.com.cn and Hao Wen, dr.wenhao@163.com

Received 7 July 2011; Accepted 28 September 2011

Academic Editor: Antonio Cascio

Copyright ( 2012 Wenbao Zhang et al. This is an open access article distributed under the Creative Commons Attribution License, which permits unrestricted use, distribution, and reproduction in any medium, provided the original work is properly cited.

Cystic echinococcosis (CE) is a cosmopolitan zoonosis caused by the larval cystic stage of the dog tapeworm Echinococcus granulosus. This complex multicellular pathogen produces various antigens which modulate the host immune response and promote parasite survival and development. The recent application of modern molecular and immunological approaches has revealed novel insights on the nature of the immune responses generated during the course of a hydatid infection, although many aspects of the Echinococcus-host interplay remain unexplored. This paper summarizes recent developments in our understanding of the immunology and diagnosis of echinococcosis, indicates areas where information is lacking, and suggests possible new strategies to improve serodiagnosis for practical application.

\section{Introduction}

Two neglected parasitic diseases, of both medical and public health importance, are cystic echinococcosis (CE) and alveolar echinococcosis (AE), caused by Echinococcus granulosus (Eg) and E. multilocularis, respectively. CE is a near-cosmopolitan zoonosis and responsible for most of the burden of echinococcosis globally [1], although $\mathrm{AE}$ is endemic in Europe [2,3] and is problematic in China [4-6].

The immunology and serodiagnosis of echinococcosis have been reviewed previously [7-10]. In this review, we summarize the general consensus of the immunology and immunodiagnosis of $\mathrm{CE}$, and reinforce previous findings with observations from some recent studies.

The Echinococcus organisms have a complex life cycle involving two hosts, a definitive carnivore host and an intermediate herbivore host. Intermediate hosts become infected by ingesting the parasite's eggs, which are released in the faeces of definitive hosts. The eggs hatch in the gastrointestinal tract and become activated larvae which penetrate the intestinal wall and enter the bloodstream, eventually locating in internal organs where they develop into hydatid cysts.
Hydatid cysts of E. granulosus develop in internal organs of humans and intermediate hosts (herbivores such as sheep, horses, cattle, pigs, goats, and camels) as unilocular fluidfilled bladders. These consist of two parasite-derived layers, an inner nucleated germinal layer and an outer acellular laminated layer surrounded by a host-produced fibrous capsule as the consequence of the host immune response [10]. Brood capsules and protoscoleces bud off from the germinal membrane. Carnivores such as dogs, wolves, and foxes act as definitive hosts. Sexual maturity of adult $E$. granulosus occurs in the host's small intestine within 4 to 5 weeks of ingesting offal containing viable protoscoleces. Gravid proglottids or released eggs are shed in the feces. An intermediate host is infected by taking an egg or eggs orally.

The intermediate host produces a significant immune response against E. granulosus infection [10]. However, the parasite has developed highly effective strategies for escaping the host defences and to avoid clearance. These mechanisms can be classified as antigenic mimicry, antigenic depletion, antigenic variation, immunologic indifference, immunologic diversion, and immunologic subversion [10]. Understanding how these immune responses are produced has been of 
fundamental importance in developing immunodiagnostic kits and highly effective recombinant vaccines against $E$. granulosus infection.

There are three significant features of E. granulosus infection: (1) the parasite uses a large number of different mammalian species as intermediate hosts. Additional species can become quickly adapted as new intermediate hosts with the production of highly fertile cysts. Examples are Australian marsupials, which have become highly susceptible to CE after E. granulosus was introduced into Australia at the time of European settlement [11], and now plays a major role in the transmission of CE on this continent $[12,13]$. (2) The resulting chronic cyst-forming disease in the intermediate host is characterized by long-term growth of the metacestode (hydatid) cysts in internal organs for as long as 53 years [14]. (3) The unilocular fluid-filled cysts can be located in most organs, with about $70 \%$ found in the liver, $20 \%$ occur in the lungs, with the remainder involving other organs such as the kidney, spleen, brain, heart, and bone. These distinct features combined with the multicellular nature of E. granulosus make $\mathrm{CE}$ a good general model for studying the immunology of chronic infections.

Cysts of E. granulosus can grow to more than $20 \mathrm{~cm}$ in diameter in humans, but the clinical manifestations are generally mild and remain asymptomatic for a considerable period. Consequently, serodiagnostic tools are important for screening populations at high risk of infection.

\section{Host Immune Responses to Hydatid Infection}

2.1. Antibody Responses. The earliest immunoglobulin (Ig) G response to CE hydatid cyst fluid and oncospheral antigens appears after 2 and 11 weeks, respectively, in mice and sheep challenged with eggs or oncospheres of E. granulosus $[15,16]$. These antioncospheral antibodies play a major role in parasite killing and are central to the protective immune response against E. granulosus [17]. Although antibody levels against the oncosphere are low [15] in the early stages of infection, the parasite killing mechanisms may involve antibody-dependent cell-mediated cytotoxicity reactions $[18,19]$.

In the chronic phases of $\mathrm{CE}$, there is frequent occurrence of elevated antibody levels, particularly IgG, IgM, and IgE [20-24], with IgG1 and IgG4 IgG subclasses being predominant $[21,25-29]$. This antibody production is essential for the development of serodiagnostic tests.

About $30-40 \%$ of patients are antibody-negative for CE. In many of these patients, however, varying levels of circulating antigens $(\mathrm{CAg})$ and circulating immune complexes (CIC) are measurable [30]. This phenomenon suggests that $\mathrm{B}$ cell activity and proliferation may be regulated and inhibited by $E$. granulosus antigens. It is not known whether these antigens directly target $\mathrm{B}$ cells or via $\mathrm{T}$ cell regulatory mechanisms.

2.2. Cellular Responses and Th2 Regulation. During the early stages of an echinococcal infection, there is a marked activation of cell-mediated immunity including cellular inflammatory responses and pathological changes [10,31]. Cellular infiltration of eosinophils, neutrophils, macrophages, and fibrocytes occurs in humans [32, 33] and sheep [34] infections. However, this generally does not result in a severe inflammatory response, and aged cysts tend to become surrounded by a fibrous layer that separates the laminated cystic layer from host tissue.

There are very few reports on $\mathrm{T}$ cell cytokine profiles in an early primary (oral challenge with eggs) E. granulosus infection. Infection with E. multilocularis eggs induced low levels of interferon- (IFN-) gama, IL-2, and IL-4 at the beginning and high levels at the end of the infection [35, $36]$, and a similar immune profile in the early stage of $\mathrm{CE}$ infection is likely.

Given the recent advances in understanding the immunoregulatory capabilities of helminthic infections, it has been suggested that Th2 responses play a crucial role in chronic helminthiasis [37]. However, a remarkable feature of chronic CE infection is the coexistence of IFN-gamma, IL-4 and IL10 at high levels in human echinococcosis [38]. It is unclear why hydatid infection can induce high levels of both Th1 and Th2 cytokines [39] since they usually downregulate each other [40]. Antigen and the amount of antigens released may play key roles. For instance, E. granulosus antigen B skewed Th1/Th2 cytokine ratios towards a preferentially immunopathology-associated Th2 polarization, predominantly in patients with progressive disease [41].

The role of IL-10 in chronic infection largely remains unclear, although one report showed that IL-10 may impair the Th1 protective response and allow the parasite to survive in hydatid patients [42]. The interaction of the Echinococcus organisms with their mammalian hosts may provide a highly suitable model to address some of the fundamental questions remaining such as the molecular basis underpinning the different effects of IL-10 on different cell types, the mechanisms of regulation of IL-10 production, the inhibitory role of IL-10 on monocyte/macrophage and CD4 $\mathrm{T}$ cell function, its involvement in stimulating the development of B cells and CD8 T cells, and its role in the differentiation and function of $\mathrm{T}$ regulatory cells.

\subsubsection{Correlation of Cytokines with Antibody Production.} Studies with mouse models to overexpress cytokines by inducing cytokine expression vectors showed that IL-12 and IFN-gamma induce a parasite-specific IgG2a response in mice infected with protoscoleces of $E$. granulosus whereas in IL-4-gene-transfected mice, IgG1 was elevated, indicating that IgG1 and IgG2 antibody isotypes are regulated by Th1 and Th2 cytokines, respectively [43].

When patients with relapsing disease or with viable, growing cysts, IgG1 and IgG4 are elevated and maintained at a high level $[21,44]$, whereas a low level of IFN-gamma produced by peripheral blood mononucleocytes (PBMC) in vitro compared with patients with a primary infection $[45,46]$. For some relapsed cases, IFN-gamma levels were undetectable in the sera of patients [47] whereas the concentrations of specific IgG1 and IgG4 declined in cases characterized by cyst infiltration or calcification [44].This indicates that the IgG4 antibody response is also associated with cystic development, growth, and disease progression 
whereas IgG1, IgG2, and IgG3 responses occur predominantly when cysts became infiltrated or are destroyed by the host [21].

2.2.2. T Cell Profile, Cyst Progression, and Efficacy of Treatment. The polarized Th2 cell response is a significant feature of the chronic stage of Echinococcus infection which is modulated by the developmental status of the hydatid cyst. In vitro $\mathrm{T}$ cell stimulation showed that cell lines from a patient with an inactive cyst had a Th1 profile while the T-cell lines derived from patients with active and transitional hydatid cysts had mixed Th1/Th2 and Th0 clones [48]. When CE patients were drug-treated with albendazole/mebendazole, a Th1 cytokine profile, rather than a Th2 profile, typically dominated, indicating that Th1 responses have a role in the process of cyst degeneration [46].

Mice injected with a vector expressing IL-4 displayed six times higher cyst load than the load in control mice [43], indicating IL-4 plays an important role in hydatid cyst development in the mammalian host.

Cytokine analysis of $177 \mathrm{CE}$ patients showed that Th1 cytokines were related to disease resistance; in contrast Th2 cytokines were associated with disease susceptibility and chronicity [38]. Both in vitro and in vivo studies have shown that high levels of the Th1 cytokine IFN-gamma were found in patients who responded to chemotherapy, whereas high levels of Th2 cytokines (IL-4 and IL-10) occurred in patients who did not [46, 49-51], indicating IL-10/IL-4 impairs the Th1 resistant response allowing E. granulosus to survive $[42,52]$.

Self-cure of CE is common in sheep [53], and it most likely also happens in human populations in hyperendemic areas as patients with calcified cysts are reported [54, 55]. It would be of value to consider the $\mathrm{T}$ cell profiles of these self-cure patients as this may impact on future treatment approaches and vaccine development.

2.2.3. Dendritic Cells. More studies have focused on dentritic cells (DC) and their regulation on other immune responses in CE. E. granulosus antigens influence maturation and differentiation of DC stimulated with lipopolysaccharide (LPS) [56]. This includes downmodulation of CD1a expression and upregulation of CD86 expression, a lower percentage of CD83(+) cells present and, downregulation of interleukin12p70 (IL-12p70) and TNF alpha [57]. In addition, hydatid cyst fluid (HCF) modulates the transition of human monocytes to DC, impairs secretion of IL-12, IL-6, or PGE2 in response to LPS stimulation, and modulates the phenotype of cells generated during culture, resulting in increased CD14 expression [56].

HCF antigen $\mathrm{B}(\mathrm{AgB})$ has been shown to induce IL-1 receptor-associated kinase phosphorylation and activate nuclear factor-kappa B, suggesting that Toll-like receptors could participate in E. granulosus-stimulated DC maturation [57].

E. multilocularis infection in mice induced DC expressing high levels TGF and very low levels of IL-10 and IL-12, and the expression of the surface markers CD80, CD86, and CD40 was downregulated $[58,59]$. However, the higher level of IL-4 than IFN-gamma/IL-2 mRNA expression in AECD4+pe-Tcells indicated DC play a role in the generation of a regulatory immune response [59].

Different E. multilocularis antigens have been shown to stimulate different expression profiles of DC. Em14-3-3antigen induced CD80, CD86, and MHC class II surface expression, but Em2(G11) failed to do so. Similarly, LPS and Em14-3-3 yielded elevated IL-12, TNF-I+/-, and IL-10 expression levels, while Em2(G11) did not. The proliferation of bone marrow DC isolated from AE-diseased mice was abrogated [60], indicating the E. multilocularis infection triggered unresponsiveness in T cells.

2.2.4. Summary of Immunological Responses in Echinococcosis and Directions for Further Study. Human helminth infections exhibit many immune downregulatory characteristics, with affected populations showing lower levels of immunopathological disease in cohort studies of allergy and autoimmunity. Model system studies have linked helminth infections with marked expansion of populations of immunoregulatory cells, such as alternatively activated macrophages, $\mathrm{T}$ regulatory cells (Tregs), and regulatory $\mathrm{B}$ cells [37].

In the established Echinococcus cystic stage, the typical response, in both humans and animals, is of the Th2 type and involves the cytokines IL-4, IL-5, IL-10, and IL-13, the antibody isotypes IgG1, IgG4, and IgE, and expanded populations of eosinophils, mast cells, and alternatively activated macrophages $[10,31]$. The precise role of Th2 responses in parasitic infections is still not very clear. It is likely that E. granulosus controls the dialogue between cells of the immune system through the release of antigens which induce Th2 responses and suppression of others involving regulatory $\mathrm{T}$ and $\mathrm{B}$ cells. Th2 is significantly associated with chronic infection and may regulate the establishment of the parasite infection. More details are needed of the regulation of Th2 cytokines on antibody production, echinococcal cyst growth, and the efficacy of treatment. The role of the antibody responses in the host parasite interaction and chronic infection remains unknown in CE.

It has been shown that in vivo depletion of DC inhibits the induction of a Th2 immune response in chronic helminth infection and DC alone can drive Th2 cell differentiation [37]. It is not known which DC signals induce the Th2 differentiation programme in naïve $\mathrm{T}$ cells [61] but $\mathrm{CE}$ represents a good model to address this issue.

As well, a number of other critical questions remain that are important for studying the role of Treg cells in the chronic infection resulting from echinococcosis such as whether Treg cells present in greater frequencies in echinococcal infections as other infections [62, 63], whether Echinococcus can expand $\mathrm{T}$ reg cell populations, and whether the parasites secrete factors which can directly induce the conversion of naïve $\mathrm{T}$ cells into functional Treg cells. There are no studies in echinococcosis on regulatory B cells, which are populations of $\mathrm{B}$ cells that downregulate immune responses. These cells are most often associated with production of the immunosuppressive cytokine IL-10. 
Moreover, many allergic and autoimmune inflammatory conditions can be ameliorated by a range of different helminth infections [64-66], so the question arises: can echinococcal infection reduce the allergic condition?

\section{Serological Diagnosis}

Typical asymptomatic features in the early stages of infection and for a long period after establishment makes early diagnosis of echinococcosis in humans difficult. Physical imaging to diagnose the CE infection, is usually used in the late stages of infection. Early diagnosis of CE by serology may, therefore, provide opportunities for early treatment and more effective chemotherapy. Another practical application of serology in human echinococcosis is the followup of the treatment.

Although hydatid disease is an asymptomatic infection, the host does produce detectable humoral and cellular responses against the infection. Measurement of these responses is a prerequisite for developing effective serodiagnostic tools.

3.1. Antibody Detection. Infection with larval cysts of Echinococcus in humans and intermediate animal hosts results in a specific antibody response, mainly of the IgG class accompanied by detectable $\operatorname{IgM}$, IgA, and IgE antibodies in some patients $[9,31,76,77]$.

In terms of methodology, almost all serological tests developed for immunodiagnosis of human CE cases have incorporated the detection of antibodies. There are considerable differences between the various tests both in specificity and sensitivity. As the sensitivity of a test increases, so generally does the demand for improved antigens in order that sufficient specificity can be achieved to take advantage of the greater sensitivity. An optimum test should be specific with high sensitivity. Insensitive and nonspecific assays including the Cassoni intradermal test, the complement fixation test (CFT), the indirect haemagglutination (IHA) test, and the latex agglutination (LA) test have been replaced by the enzyme-linked immunosorbent assay (ELISA), the indirect immunofluorescence antibody test (IFAT), immunoelectrophorosis (IEP), and immunoblotting (IB) in routine laboratory application [78].

A comparison of the diagnostic sensitivity and specificity of IEP, ELISA, and IB, in detecting IgG antibodies in patient sera to native and recombinant $\mathrm{AgB}$ and a hydatid fluid fraction (HFF), showed that HFF-IB gave the highest sensitivity (80\%) followed by ELISA (72\%) and IEP $(31 \%)$. The diagnostic sensitivity significantly decreased as cysts matured (from type I-II to type VII, classified by ultrasound). Recombinant and native AgB-IB yielded similar levels of sensitivity (74\%) but a large number of clinically or surgically confirmed CE patients (20\%) were negative. In these patient sera, IB, to assess the usefulness of another recombinant E. granulosus molecule (elongation factor-1 beta/delta) in detecting IgE antibodies, yielded a positivity of $33 \%$. Serological tests developed for determining antiEchinococcus IgE in serum usually express results qualitatively or semiquantitatively in titres or units specific for the test kit $[20,79,80]$.

The serodiagnostic performance of a range of different antigens and the various methods available for immunodiagnosis have been reviewed in depth [10, 31]. Some recent studies are referred to in Table 1 with the sensitivity and specificity of individual tests listed. Some antigens, such as native $\mathrm{AgB}$ and its recombinant proteins, yielded reasonable diagnostic performance using panels of sera from clinically confirmed cases of echinococcosis and other helminth infections. However, when the antigens were used for screening human populations in hyperendemic communities, they showed high seropositivity rates, although these rates had a low correlation with US monitoring of individual subjects [81].

Recently developed dipstick assays [82] are considered to be valuable methods for $\mathrm{CE}$ serodiagnosis. One dipstick assay has been developed that exhibited $100 \%$ sensitivity and 91.4\% specificity when tested on sera from 26 CE patients and sera from 35 subjects with other parasitic infections using camel hydatid cyst fluid as antigen [83]. Since the dipstick assay is extremely easy to perform with a visually interpretable result within $15 \mathrm{~min}$, in addition to being both sensitive and specific, the test could be an acceptable alternative for use in clinical laboratories lacking specialized equipment or the technological expertise needed for western blotting or ELISA. Similarly, a new 3-minute rapid dot immunogold filtration assay (DIGFA) for serodiagnosis of human $\mathrm{CE}$ and $\mathrm{AE}$ has been developed using four native antigen preparations crude and partially purified hydatid cyst fluid extracts from E. granulosus ( $\mathrm{EgCF}$ and $\mathrm{AgB}$ ), E. granulosus protoscolex extract (EgP), and E. multilocularis metacestode antigen (Em2) [70]. Like the dipstick assay, the test incorporates a simple eye-read colour change and achieved an overall sensitivity of $80.7 \%$ for human CE and 92.9\% for human $\mathrm{AE}$ in a hospital diagnostic setting [70]. These rapid test scan be used for both clinical diagnostic support, as well as combining with ultrasound for mass screening in areas endemic for $\mathrm{CE}$ and $\mathrm{AE}$.

Standardization of techniques and antigenic preparations and the characterization of new antigens are urgently required to improve the performance of hydatid immunodiagnosis. Antigens used in current tests are either cyst fluid or crude homogenates of the parasite collected from domestic animals. However, the supply of antigenic sources can often be limited, even for laboratory use. Since the preparation of purified echinococcal antigens relies on the availability of parasitic material and the quality control of this material is difficult to standardize for a large scale production, this can impact substantially on sensitivity and specificity of the available immunodiagnostic tools.

3.2. Antigen Detection. Antibody detection is likely to indicate exposure to an Echinococcus infection, but it may not necessarily indicate the presence of an established and viable infection, or the disease. Serum antibodies may persist for a prolonged period, reaching up to 10 years after hydatid cyst removal [84]. In addition, the degree of antibody response may be related to the location and condition of a mature 
TABle 1: Characteristics of assays using different antigens from E. granulosus developed after 2003 for immunodiagnosis of cystic echinococcosis.

\begin{tabular}{|c|c|c|c|c|c|c|c|c|}
\hline \multicolumn{3}{|c|}{ Number of subjects tested } & \multirow{2}{*}{ Antigen } & \multirow{2}{*}{ Assay method } & \multirow{2}{*}{ Sensitivity (\%) } & \multirow{2}{*}{ Specificity (\%) } & \multirow{2}{*}{ Ig isotype } & \multirow{2}{*}{ Refs. } \\
\hline $\mathrm{CE}$ & Healthy controls & Other diseases & & & & & & \\
\hline 44 & - & 43 & $8 \mathrm{kDa}$ & WB & 47.7 & 51.2 & $\operatorname{IgG}$ & {$[67]$} \\
\hline 44 & - & 43 & $16 \mathrm{kDa}$ & WB & 45.5 & 67.4 & IgG & {$[67]$} \\
\hline 44 & - & 43 & $24 \mathrm{kDa}$ & WB & 68.2 & 62.8 & IgG & {$[67]$} \\
\hline 36 & 36 & - & $\mathrm{AgB}$ & ELISA & 91.7 & 97.2 & $\operatorname{IgG}$ & {$[68]$} \\
\hline 102 & 95 & 68 & $\mathrm{rAgB1}$ & ELISA & 88.2 & 80.9 & IgG & [69] \\
\hline 102 & 95 & 68 & $\mathrm{rAgB} 2$ & ELISA & 91.2 & 93 & IgG & [69] \\
\hline 875 & 5 & 739 & $\mathrm{AgB}$ & Dot-WB & 68.4 & 93.4 & IgG & {$[70]$} \\
\hline 857 & 5 & 739 & $\mathrm{AgB}$ & ELISA & 57.4 & 93.4 & $\operatorname{IgG}$ & {$[70]$} \\
\hline 324 & 70 & 500 & $\mathrm{AB}$ & WB & 86.4 & 92 & IgG & [71] \\
\hline 155 & 110 & 58 & $?$ & ELISA & 73.6 & 99.1 & $\operatorname{IgE}$ & [72] \\
\hline 875 & 5 & 739 & $\mathrm{AgB}$ & Dot-WB & 68.4 & 93.4 & $\operatorname{IgG}$ & [70] \\
\hline 857 & 5 & 739 & $\mathrm{AgB}$ & ELISA & 57.4 & 93.4 & IgG & [70] \\
\hline 324 & 70 & 500 & $\mathrm{AB}$ & WB & 86.4 & 92 & IgG & [71] \\
\hline 155 & 110 & 58 & $?$ & ELISA & 73.6 & 99.1 & $\operatorname{IgE}$ & [72] \\
\hline 155 & 110 & 58 & $?$ & ELISA & 90.3 & 90.9 & IgG & [72] \\
\hline 155 & 110 & 58 & $\mathrm{HCF}$ & WB & 90.1 & 94.5 & IgG & [72] \\
\hline 324 & 70 & 500 & $\mathrm{EpC1}$ & WB & 88.7 & 95.6 & $\operatorname{IgG}$ & [71] \\
\hline 95 & 37 & - & HSP20 & & 64 & & $\operatorname{IgG} 1,4$ & [73] \\
\hline 97 & 37 & 58 & Eg19 & WB & 10 & 100 & $\operatorname{IgG}$ & [74] \\
\hline 102 & 95 & 68 & E14t & ELISA & 35.3 & 91.7 & IgG & [69] \\
\hline 102 & 95 & 68 & C317 & ELISA & 58.8 & 80.9 & $\operatorname{IgG}$ & [69] \\
\hline 60 & - & - & P5 & WB & 97 & 1 & 1 & [75] \\
\hline
\end{tabular}

ELISA: enzyme-linked immunosorbent assay; WB: western blotting; dELISA: dot enzyme-linked immunosorbent assay.

hydatid cyst. For instance, hydatid cysts in human lung, spleen, or kidney tend to be associated with lower serum antibody levels [9]. Furthermore, in Echinococcus-endemic villages, up to $26 \%$ or more of the general population may have antibodies to HCF antigens, but with only about $2 \%$ of the villagers having hydatid cysts $[81,85,86]$, indicating that the antibody levels may not necessarily reflect the true prevalence of CE.

Antigen detection may provide a suitable alternative. Serum antigen detection may also be less affected by hydatid cyst location and provides a tool for serological monitoring of antiparasitic therapy [87]. Circulating antigen (CAg) in CE patient sera, can be detected using ELISA directly or indirectly, and against titrated cyst fluid standards, CAg concentrations have been shown to vary from 100 to $700 \mathrm{ng} / \mathrm{mL}$ [88].

Antigen detection assays depend principally on the binding of specific polyclonal or monoclonal antibodies to parasite antigen present in serum or urine. A number of different assays have been developed to detect echinococcal antigens. The standard double antibody sandwich ELISA is a common method for measuring the presence and/or concentration of circulating parasite antigens. In the test, antibody raised to the targeted protein is coated onto a microtiter plate to capture antigen (Figure 1). The same antibody, which is enzyme labelled, is commonly used in the tertiary layer of the assay. This type of antigen capture therefore relies on the presence of multiple binding sites on the target antigens(s). Efforts to detect CAg in CE patients have been reviewed extensively by Craig et al. [85].

CAg in serum is normally in the form of a circulating immune complex (CIC) with some in free form. Therefore, the serum needs to be treated with acid buffer or polyethylene glycol (PEG) to release and concentrate the circulating antigens. Acidic treatment $(0.2 \mathrm{M}$ glycine $/ \mathrm{HCl})$ of $\mathrm{CE}$ patient serum is quite straightforward to dissociate CIC [85]. In a comparison of acid-treatment and PEG precipitation methods, all the sera of 30 confirmed positive cases of CE had detectable levels of antigen in the acidtreated sera [30]. However, $23(77 \%)$ and $26(87 \%)$ sera of 30 confirmed cases had free antigen as well as CIC of an $8 \mathrm{kDa}$ antigen in the untreated and in the polyethylene glycol (PEG) precipitated sera, respectively. None of the sera from other patients with parasitic infections or viral hepatitis had any detectable levels of $8 \mathrm{kDa}$ antigen in the untreated, acid-treated, or PEG-precipitated serum samples. These 
(1)

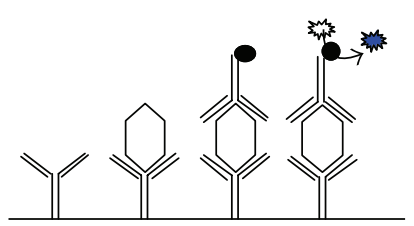

(a)

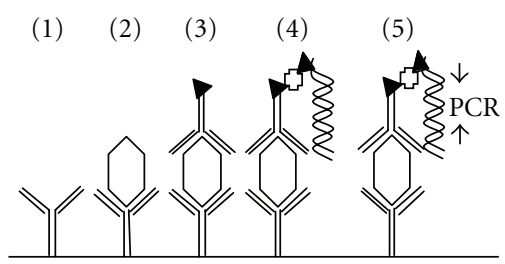

(c)

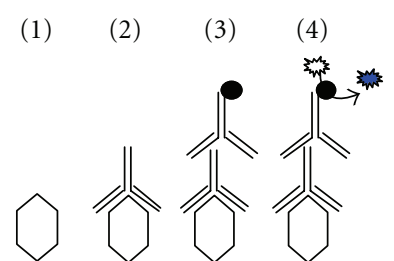

(b)

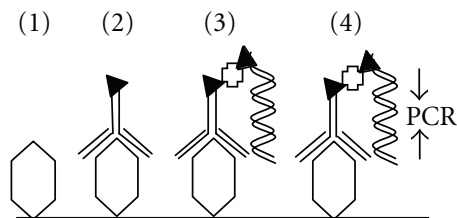

(d)

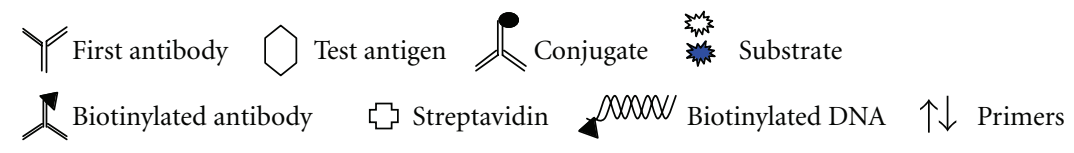

FIGURE 1: Schematic of ELISA and immuno-PCR for detecting circulating antigen in serum. (a) Sandwich ELISA. (1) Plate is coated with a capture antibody; (2) serum sample is added, and any antigen present in the serum binds to the capture antibody; (3) detecting antibody conjugate is added and binds to the antigen; (4) substrate is added, and is converted by the enzyme to a detectable form. (b) Direct ELISA. Plate is coated with diluted serum containing antigen; (2) detecting antibody is added, and binds to antigen; (3) enzyme-linked secondary antibody is added, and binds to detecting antibody; (4) substrate is added and is converted by the enzyme to a detectable form. (c) Capture immuno-PCR. (1) Plate is coated with capture antibody; (2) serum sample is added; (3) biotinylated detecting antibody is added and binds to antigen; (4) Streptavidin and biotinylated reporter DNA are added, and the biotinylated antibody and biotinylated reporter DNA are linked by streptavidin; (5) Primers and PCR components are added and PCR or real-time PCR undertaken to quantify antigen. (d) Non-capture immuno-PCR. Serum sample is coated on the plate and the remainder of the steps are as for the capture-immuno-PCR (C).

investigations, therefore, suggested that the demonstration of circulating antigen employing monospecific antibodies to affinity purified $8 \mathrm{kDa}$ antigen in acid-treated sera is more efficient than the detection of free circulating antigen or CIC in untreated or in PEG-precipitated sera [89].

IgM CICs tend to be positively associated with active hydatid disease $[85,90]$. Combining measurement of circulating antibody, CICs, and CAg resulted in an increase from $77 \%$ to $90 \%$ compared to measurement of serum antibody alone [91]. Antigens in soluble CICs from CE patients have been characterized by separating them on SDS-PAGE [85] or by ion-exchange fast protein liquid chromatography (FPLC) [92]. Both studies indicated a candidate antigen detectable in serum with an approximate relative molecular mass of 60 $67 \mathrm{KDa}$, and which is also present in cyst fluid.

Comparison of CAg and IgG antibody using ELISA, together with western blotting, showed a relatively low sensitivity (43\%) for detection of specific serum antigen in $\mathrm{CE}$, compared to $75 \%$ for IgG antibodies [93]. However, the specificity of this CAg ELISA was $90 \%$ when tested against sera from $\mathrm{AE}$ patients and $100 \%$ against human cysticercosis sera. The limited cross-reactivity may be a way for practical diagnosis of $\mathrm{CE}$ in areas where $\mathrm{AE}$ and cysticercosis are coendemic. The advantage of CAg detection is its high sensitivity for detecting CE in $54-57 \%$ of patients who are serum antibody negative $[91,93]$. CAg detection does appear, therefore, to be potentially useful as a secondary test for some suspected CE cases where antibody titers are low $[85,94]$.
A combination of CAg and antibody detection has been shown to increase the sensitivity from $85 \%$ (antibody only) to $89 \%$ (antibody+CAg) in ELISA of 115 surgically confirmed hydatid patients, 41 individuals exhibiting other parasitic and unrelated diseases, and 69 healthy subjects [95].

Although there has been no application to date for echinococcal diagnosis, a technique for antigen detection, called immunopolymerase chain reaction (immuno-PCR), was developed by Sano et al. [96]. It combines the molecular recognition of antibodies with the high DNA amplification capability of PCR. The procedure is similar to conventional ELISA but is far more sensitive. And, in principle, could be applied to the detection of single antigen molecules. Instead of an enzyme, a DNA molecule is linked to the detection antibody and serves as a template for PCR (Figure 1). The DNA molecule is amplified and the PCR product is measured by gel electrophoresis. An improvement of this method is to amplify the DNA fragment by real-time PCR, thereby eliminating post-PCR analysis. Furthermore, real-time PCR is extremely accurate and sensitive, which should make it possible to quantitate very low amounts of DNA-coupled detection antibody with high accuracy.

3.3. Serodiagnosis: The Future. Almost all available immunodiagnostic techniques, including methods for detecting specific antibodies and circulating parasite antigens in serum or other body fluids, have been applied for diagnosing echinococcosis. However, all the tools developed to date are 
generally applicable for laboratory research purposes only. None of the available diagnostic tools, kits, or methods are generally accepted by clinical physicians. Nevertheless, such serological tools are potentially important for epidemiological studies, confirmation of infection status, and treatment and the monitoring of control programs, and efforts should continue so that new assays for improved, practical diagnosis of echinococcosis are developed.

\section{References}

[1] C. M. Budke, "Global socioeconomic impact of cystic echinococcosis," Emerging Infectious Diseases, vol. 12, no. 2, pp. 296303, 2006.

[2] P. Deplazes, "Ecology and epidemiology of Echinococcus multilocularis in Europe," Parassitologia, vol. 48, no. 1-2, pp. 37-39, 2006.

[3] J. Eckert, F. J. Conraths, and K. Tackmann, "Echinococcosis: an emerging or re-emerging zoonosis?" International Journal for Parasitology, vol. 30, no. 12-13, pp. 1283-1294, 2000.

[4] T. Li, X. Chen, R. Zhen et al., "Widespread co-endemicity of human cystic and alveolar echinococcosis on the eastern Tibetan Plateau, northwest Sichuan/southeast Qinghai, China," Acta Tropica, vol. 113, no. 3, pp. 248-256, 2010.

[5] Y. R. Yang, G. M. Williams, P. S. Craig et al., "Hospital and community surveys reveal the severe public health problem and socio-economic impact of human echinococcosis in Ningxia Hui Autonomous Region, China," Tropical Medicine and International Health, vol. 11, no. 6, pp. 880-888, 2006.

[6] P. S. Craig, "Epidemiology of human alveolar echinococcosis in China," Parasitology International, vol. 55, supplement 1, pp. S221-S225, 2006.

[7] A. Siracusano, R. Rigano, E. Ortona et al., "Immunomodulatory mechanisms during Echinococcus granulosus infection," Experimental Parasitology, vol. 119, no. 4, pp. 483-489, 2008.

[8] D. Carmena, A. Benito, and E. Eraso, "Antigens for the immunodiagnosis of Echinococcus granulosus infection: an update," Acta Tropica, vol. 98, no. 1, pp. 74-86, 2006.

[9] W. Zhang and D. P. McManus, "Recent advances in the immunology and diagnosis of echinococcosis," FEMS Immunology and Medical Microbiology, vol. 47, no. 1, pp. 2441, 2006.

[10] W. Zhang, J. Li, and D. P. McManus, "Concepts in immunology and diagnosis of hydatid disease," Clinical Microbiology Reviews, vol. 16, no. 1, pp. 18-36, 2003.

[11] D. J. Jenkins and C. N. L. Macpherson, "Transmission ecology of Echinococcus in wild-life in Australia and Africa," Parasitology, vol. 127, supplement 1, pp. S63-S72, 2003.

[12] T. S. Barnes, J. M. Morton, and G. T. Coleman, "Clustering of hydatid infection in macropodids," International Journal for Parasitology, vol. 37, no. 8-9, pp. 943-952, 2007.

[13] D. J. Jenkins, "Echinococcus granulosus in Australia, widespread and doing well!," Parasitology International, vol. 55, supplement 1, pp. S203-S206, 2006.

[14] S. L. Spruance, "Latent period of 53 years in a case of hydatid cyst disease," Archives of Internal Medicine, vol. 134, no. 4, pp. 741-742, 1974.

[15] W. Zhang, H. You, J. Li et al., "Immunoglobulin profiles in a murine intermediate host model of resistance for Echinococcus granulosus infection," Parasite Immunology, vol. 25, no. 3, pp. 161-168, 2003.

[16] W. K. Yong, D. D. Heath, and F. Van Knapen, "Comparison of cestode antigens in an enzyme-linked immunosorbent assay for the diagnosis of Echinococcus granulosus, Taenia hydatigena and T ovis infections in sheep," Research in Veterinary Science, vol. 36, no. 1, pp. 24-31, 1984.

[17] R. P. Dempster, G. B. L. Harrison, M. V. Berridge, and D. D. Heath, "Echinococcus granulosus: use of an intermediate host mouse model to evaluate sources of protective antigens and a role for antibody in the immune response," International Journal for Parasitology, vol. 22, no. 4, pp. 435-441, 1992.

[18] P. L. Beardsell and M. J. Howell, "Killing of Taenia hydatigena oncospheres by sheep neutrophils," Zeitschrift fur Parasitenkunde, vol. 70, no. 3, pp. 337-344, 1984.

[19] M. T. Rogan, P. S. Craig, E. Zehyle, G. Masinde, H. Wen, and P. Zhou, "In vitro killing of taeniid oncospheres, mediated by human sera from hydatid endemic areas," Acta Tropica, vol. 51, no. 3-4, pp. 291-296, 1992.

[20] A. R. Khabiri, F. Bagheri, M. Assmar, and M. R. Siavashi, "Analysis of specific IgE and IgG subclass antibodies for diagnosis of Echinococcus granulosus," Parasite Immunology, vol. 28, no. 8, pp. 357-362, 2006.

[21] A. O. Daeki, P. S. Craig, and M. K. Shambesh, "IgG-subclass antibody responses and the natural history of hepatic cystic echinococcosis in asymptomatic patients," Annals of Tropical Medicine and Parasitology, vol. 94, no. 4, pp. 319-328, 2000.

[22] J. M. Pinon, J. Poirriez, H. Lepan, R. Geers, R. Penna, and D. Fernandez, "Value of isotypic characterization of antibodies to Echinococcus granulosus by enzyme-linked immuno-filtration assay," European Journal of Clinical Microbiology, vol. 6, no. 3, pp. 291-295, 1987.

[23] P. S. Craig, "Detection of specific circulating antigen, immune complexes and antibodies in human hydatidosis from Turkana (Kenya) and Great Britain, by enzyme-immunoassay," Parasite Immunology, vol. 8, no. 2, pp. 171-188, 1986.

[24] J. P. Dessaint, D. Bout, P. Wattre, and A. Capron, "Quantitative determination of specific IgE antibodies to Echinococcus granulosus and IgE levels in sera from patients with hydatid disease," Immunology, vol. 29, no. 5, pp. 813-823, 1975.

[25] S. Sterla, H. Sato, and A. Nieto, "Echinococcus granulosus human infection stimulates low avidity anticarbohydrate IgG2 and high avidity antipeptide IgG4 antibodies," Parasite Immunology, vol. 21, no. 1, pp. 27-34, 1999.

[26] A. Aceti, A. Pennica, A. Teggi et al., "IgG subclasses in human hydatid disease: prominence of the IgG4 response," International Archives of Allergy and Immunology, vol. 102, no. 4, pp. 347-351, 1993.

[27] H. Wen and P. S. Craig, "Immunoglobulin G subclass responses in human cystic and alveolar echinococcosis," American Journal of Tropical Medicine and Hygiene, vol. 51, no. 6, pp. 741-748, 1994.

[28] M. K. Shambesh, P. S. Craig, H. Wen, M. T. Rogan, and E. Paolillo, "IgG1 and IgG4 serum antibody responses in asymptomatic and clinically expressed cystic echinococcosis patients," Acta Tropica, vol. 64, no. 1-2, pp. 53-63, 1997.

[29] S. Ioppolo, S. Notargiacomo, E. Profumo et al., "Immunological responses to antigen B from Echinococcus granulosus cyst fluid in hydatid patients," Parasite Immunology, vol. 18, no. 11, pp. 571-578, 1996.

[30] P. S. Craig, "Immunodiagnosis of Echinococcus granulosus," in Compendium on Cystic Echinococcosis with Special Reference, $\mathrm{F}$. L. Andersen, J.-J. Chai, and F.-J. Liu, Eds., pp. 85-118, Brigham Young University, Provo, Utah, USA, 1993.

[31] W. Zhang, A. G. Ross, and D. P. McManus, "Mechanisms of immunity in hydatid disease: implications for vaccine development," Journal of Immunology, vol. 181, no. 10, pp. 6679-6685, 2008. 
[32] X. Peng, J. Li, X. Wu et al., "Detection of Osteopontin in the pericyst of human hepatic Echinococcus granulosus," Acta Tropica, vol. 100, no. 3, pp. 163-171, 2006.

[33] J. K. Magambo, , , J, Raasen T, Zeyhle, T. M. Wachira, J. Wachira, and T. Raasen, "Cellular immunity to Echinococcus granulosus cysts," African Journal of Health Sciences, vol. 2, pp. 250-253, 1995.

[34] R. F. Petrova, "Blood picture in experimental hydatidosis in sheep," Zhivotnikh v Chimkente, pp. 115-116, 1968 (Russian).

[35] B. Bauder, H. Auer, F. Schilcher et al., "Experimental investigations on the $\mathrm{B}$ and $\mathrm{T}$ cell immune response in primary alveolar echinococcosis," Parasite Immunology, vol. 21, no. 8, pp. 409421, 1999.

[36] D. A. Vuitton, "The ambiguous role of immunity in echinococcosis: protection of the host or of the parasite?" Acta Tropica, vol. 85, no. 2, pp. 119-132, 2003.

[37] J. E. Allen and R. M. Maizels, "Diversity and dialogue in immunity to helminths," Nature Reviews Immunology, vol. 11, pp. 375-388, 2011.

[38] D. Mezioug and C. Touil-Boukoffa, "Cytokine profile in human hydatidosis: possible role in the immunosurveillance of patients infected with Echinococcus granulosus," Parasite, vol. 16, no. 1, pp. 57-64, 2009.

[39] R. Riganò, E. Profumo, G. Di Felice, E. Ortona, A. Teggi, and A. Siracusano, "In vitro production of cytokines by peripheral blood mononuclear cells from hydatid patients," Clinical and Experimental Immunology, vol. 99, pp. 433-439, 1995.

[40] E. J. Pearce and A. S. MacDonald, "The immunobiology of schistosomiasis," Nature Reviews Immunology, vol. 2, no. 7, pp. 499-511, 2002.

[41] R. Riganò, E. Profumo, F. Bruschi et al., "Modulation of human immune response by Echinococcus granulosus antigen $\mathrm{B}$ and its possible role in evading host defenses," Infection and Immunity, vol. 69, no. 1, pp. 288-296, 2001.

[42] M. Amri, D. Mezioug, and C. Touil-Boukoffa, "Involvement of IL-10 and IL-4 in evasion strategies of Echinococcus granulosus to host immune response," European Cytokine Network, vol. 20, no. 2, pp. 63-68, 2009.

[43] K. M. Al-Qaoud and S. K. Abdel-Hafez, "The induction of T helper type 1 response by cytokine gene transfection protects mice against secondary hydatidosis," Parasitology Research, vol. 102, no. 6, pp. 1151-1155, 2008.

[44] M. R. Bayraktar, N. Mehmet, and R. Durmaz, "Th1 and Th2 inducing cytokines in Cystic echinococcosis," Turkiye Parazitol Derg , vol. 29, pp. 167-170, 2005.

[45] R. Rigano, E. Profumo, A. Teggi, and A. Siracusano, "Production of IL- 5 and IL-6 by peripheral blood mononuclear cells (PBMC) from patients with Echinococcus granulosus infection," Clinical and Experimental Immunology, vol. 105, no. 3, pp. 456-459, 1996.

[46] R. Rigano, E. Profumo, S. Ioppolo et al., "Immunological markers indicating the effectiveness of pharmacological treatment in human hydatid disease," Clinical and Experimental Immunology, vol. 102, no. 2, pp. 281-285, 1995.

[47] C. Touil-Boukoffa, J. Sanceau, B. Tayebi, and J. Wietzerbin, "Relationship among circulating interferon, tumor necrosis factor-alpha, and interleukin- 6 and serologic reaction against parasitic antigen in human hydatidosis," Journal of Interferon and Cytokine Research, vol. 17, no. 4, pp. 211-217, 1997.

[48] R. Rigano, B. Buttari, E. De Falco et al., "Echinococcus granulosus-specific T-cell lines derived from patients at various clinical stages of cystic echinococcosis," Parasite Immunology, vol. 26, no. 1, pp. 45-52, 2004.
[49] R. Rigano, E. Profumo, S. Ioppolo, S. Notargiacomo, A. Teggi, and A. Siracusano, "Serum cytokine detection in the clinical follow up of patients with cystic echinococcosis," Clinical and Experimental Immunology, vol. 115, no. 3, pp. 503-507, 1999.

[50] R. Rigano, E. Profumo, B. Buttari, A. Teggi, and A. Siracusano, "Cytokine gene expression in peripheral blood mononuclear cells (PBMC) from patients with pharmacologically treated cystic echinococcosis," Clinical and Experimental Immunology, vol. 118, no. 1, pp. 95-101, 1999.

[51] R. Rigano, E. Profumo, G. Di Felice, E. Ortona, A. Teggi, and A. Siracusano, "In vitro production of cytokines by peripheral blood mononuclear cells from hydatic patients," Clinical and Experimental Immunology, vol. 99, no. 3, pp. 433-439, 1995.

[52] A. Siracusano, F. Delunardo, A. Teggi et al., "Host-parasite relationship in cystic echinococcosis: an evolving story," Clinical and Developmental Immunology, Article ID 639362, 12 pages, 2012.

[53] P. A. Cabrera, P. Irabedra, D. Orlando et al., "National prevalence of larval echinococcosis in sheep in slaughtering plants Ovis aries as an indicator in control programmes in Uruguay," Acta Tropica, vol. 85, no. 2, pp. 281-285, 2003.

[54] P. L. Moro, H. H. Garcia, A. E. Gonzales, J. J. Bonilla, M. Verastegui, and R. H. GilmanMD, "Screening for cystic echinococcosis in an endemic region of Peru using portable ultrasonography and the enzyme-linked immunoelectrotransfer blot (EITB) assay," Parasitology Research, vol. 96, no. 4, pp. 242-246, 2005.

[55] C. N. L. Macpherson, M. Kachani, M. Lyagoubi et al., "Cystic echinococcosis in the Berber of the Mid Atlas mountains, Morocco: vew insights into the natural history of the disease in humans," Annals of Tropical Medicine and Parasitology, vol. 98, no. 5, pp. 481-490, 2004.

[56] J. H. C. Kanan and B. M. Chain, "Modulation of dendritic cell differentiation and cytokine secretion by the hydatid cyst fluid of Echinococcus granulosus," Immunology, vol. 118, no. 2, pp. 271-278, 2006.

[57] R. Rigano, B. Buttari, E. Profumo et al., "Echinococcus granulosus antigen B impairs human dendritic cell differentiation and polarizes immature dendritic cell maturation towards a Th2 cell response," Infection and Immunity, vol. 75, no. 4, pp. 1667-1678, 2007.

[58] N. Mejri, N. Muller, A. Hemphill, and B. Gottstein, "Intraperitoneal Echinococcus multilocularis infection in mice modulates peritoneal CD4+ and CD8+ regulatory T cell development," Parasitology International, vol. 60, pp. 45-53, 2011.

[59] N. Mejri, J. Muller, and B. Gottstein, "Intraperitoneal murine Echinococcus multilocularis infection induces differentiation of TGF-beta expressing DCs that remain immature," Parasite Immunology, vol. 33, no. 9, pp. 471-482, 2011.

[60] M. C. Margos, D. Grandgirard, S. Leib, and B. Gottstein, "In vitro induction of lymph node cell proliferation by mouse bone marrow dendritic cells following stimulation with different Echinococcus multilocularis antigens," Journal of Helminthology, vol. 85, no. 2, pp. 128-137, 2011.

[61] A. S. MacDonald and R. M. Maizels, "Alarming dendritic cells for Th2 induction," Journal of Experimental Medicine, vol. 205, no. 1, pp. 13-17, 2008.

[62] C. A. M. Finney, M. D. Taylor, M. S. Wilson, and R. M. Maizels, "Expansion and activation of $\mathrm{CD} 4(+) \mathrm{CD} 25(+)$ regulatory $\mathrm{T}$ cells in Heligmosomoides polygyrus infection," European Journal of Immunology, vol. 37, no. 7, pp. 1874-1886, 2007.

[63] S. Rausch, J. Huehn, D. Kirchhoff et al., "Functional analysis of effector and regulatory $\mathrm{T}$ cells in a parasitic nematode 
infection," Infection and Immunity, vol. 76, no. 5, pp. 1908 1919, 2008.

[64] D. E. Elliott, R. W. Summers, and J. V. Weinstock, "Helminths as governors of immune-mediated inflammation," International Journal for Parasitology, vol. 37, no. 5, pp. 457-464, 2007.

[65] R. M. Maizels, "Infections and allergy-helminths, hygiene and host immune regulation," Current Opinion in Immunology, vol. 17, no. 6, pp. 656-661, 2005.

[66] P. G. Fallon and N. E. Mangan, "Suppression of TH2-type allergic reactions by helminth infection," Nature Reviews Immunology, vol. 7, no. 3, pp. 220-230, 2007.

[67] M. L. de la Rue, K. Yamano, C. E. Almeida et al., "Serological reactivity of patients with Echinococcus infections (E. granulosus, E. vogeli, and E. multilocularis) against three antigen B subunits," Parasitology Research, vol. 106, no. 3, pp. 741-745, 2010.

[68] E. Kalantari, M. Bandehpour, R. Pazoki et al., "Application of recombinant Echinococcus granulosus antigen B to ELISA kits for diagnosing hydatidosis," Parasitology Research, vol. 106, no. 4, pp. 847-851, 2010.

[69] A. Hernandez-Gonzalez, A. Muro, I. Barrera, G. Ramos, A. Orduna, and M. Siles-Lucas, "Usefulness of four different Echinococcus granulosus recombinant antigens for serodiagnosis of unilocular hydatid disease (UHD) and postsurgical follow-up of patients treated for UHD," Clinical and Vaccine Immunology, vol. 15, no. 1, pp. 147-153, 2008.

[70] X. Feng, H. Wen, Z. Zhang et al., "Dot immunogold filtration assay (DIGFA) with multiple native antigens for rapid serodiagnosis of human cystic and alveolar echinococcosis," Acta Tropica, vol. 113, no. 2, pp. 114-120, 2010.

[71] J. Li, W. B. Zhang, M. Wilson, A. Ito, and D. P. McManus, "A novel recombinant antigen for immunodiagnosis of human cystic echinococcosis," Journal of Infectious Diseases, vol. 188, no. 12, pp. 1952-1960, 2003.

[72] I. Marinova, G. Nikolov, A. Michova, R. Kurdova, and B. Petrunov, "Quantitative assessment of serum-specific IgE in the diagnosis of human cystic echinococcosis," Parasite Immunology, vol. 33, no. 7, pp. 371-376, 2011.

[73] D. Vacirca, M. Perdicchio, E. Campisi et al., "Favourable prognostic value of antibodies anti-HSP20 in patients with cystic echinococcosis: a differential immunoproteomic approach," Parasite Immunology, vol. 33, no. 3, pp. 193-198, 2011.

[74] F. Delunardo, E. Ortona, P. Margutti et al., "Identification of a novel $19 \mathrm{kDa}$ Echinococcus granulosus antigen," Acta Tropica, vol. 113, no. 1, pp. 42-47, 2010.

[75] W. B. Zhang, J. Li, Q. Li et al., "Identification of a diagnostic antibody-binding region on the immunogenic protein EpC1 from Echinococcus granulosus and its application in population screening for cystic echinococcosis," Clinical and Experimental Immunology, vol. 149, no. 1, pp. 80-86, 2007.

[76] D. A. Vuitton and B. Gottstein, "Echinococcus multilocularis and its intermediate host: a model of parasite-host interplay," Journal of Biomedicine and Biotechnology, vol. 2010, Article ID 923193, 2010.

[77] Y. Sbihi, D. Janssen, and A. Osuna, "Specific recognition of hydatid cyst antigens by serum IgG, IgE, and IgA using Western blot," Journal of Clinical Laboratory Analysis, vol. 11, no. 3, pp. 154-157, 1997.

[78] M. A. Nasrieh and S. K. Abdel-Hafez, "Echinococcus granulosus in Jordan: assessment of various antigenic preparations for use in the serodiagnosis of surgically confirmed cases using enzyme immuno assays and the indirect haemagglutination test," Diagnostic Microbiology and Infectious Disease, vol. 48, no. 2, pp. 117-123, 2004.
[79] B. Saenz de San Pedro, J. L. Cazana, J. Cobo et al., "Anaphylactic shock by rupture of hydatid heptic cyst. Follow-up by specific IgE serum antibodies," Allergy, vol. 47, no. 5, pp. 568570, 1992.

[80] M. Chamekh, H. Gras-Masse, M. Bossus et al., "Diagnostic value of a synthetic peptide derived from Echinococcus granulosus recombinant protein," Journal of Clinical Investigation, vol. 89, no. 2, pp. 458-464, 1992.

[81] C. M. Gavidia, A. E. Gonzalez, W. Zhang et al., "Diagnosis of cystic echinococcosis, central peruvian highlands," Emerging Infectious Diseases, vol. 14, no. 2, pp. 260-266, 2008.

[82] H. R. van Doorn, R. Koelewijn, H. Hofwegen et al., "Use of enzyme-linked immunosorbent assay and dipstick assay for detection of strongyloides stercoralis infection in humans," Journal of Clinical Microbiology, vol. 45, no. 2, pp. 438-442, 2007.

[83] M. M. Al-Sherbiny, A. A. M. Farrag, M. H. Fayad, M. K. Makled, G. M. Tawfeek, and N. M. S. Ali, "Application and assessment of a dipstick assay in the diagnosis of hydatidosis and trichinosis," Parasitology Research, vol. 93, no. 2, pp. 8795, 2004.

[84] J. Li, W. B. Zhang, and D. P. McManus, "Recombinant antigens for immunodiagnosis of cystic echinococcosis," Biological Procedures Online, vol. 6, no. 1, pp. 67-77, 2004.

[85] P. S. Craig, E. Zeyhle, and T. Romig, "Hydatid disease: research and control in Turkana. II. The role of immunological techniques for the diagnosis of hydatid disease," Royal Society of Tropical Medicine and Hygiene, vol. 80, pp. 183-192, 1986.

[86] J. Chai, Y. Sultan, and M. Wei, "An investigation on the epidemiologic baseline of hydatid disease in Xinjiang, China. I. A sero-epidemiological survey of human hydatidosis," Endemic Diseases Bulletin, vol. 4, pp. 1-8, 1989 (Chinese).

[87] P. S. Craig and G. S. Nelson, "The detection of circulating antigen in human hydatid disease," Annals of Tropical Medicine and Parasitology, vol. 78, no. 3, pp. 219-227, 1984.

[88] B. Gottstein, "An immunoassay for the detection of circulating antigens in human echinococcosis," American Journal of Tropical Medicine and Hygiene, vol. 33, no. 6, pp. 1185-1191, 1984.

[89] J. R. Kanwar, R. K. Kanwar, A. S. Grewal, and V. K. Vinayak, "Significance of detection of immune-complexed $8 \mathrm{kDa}$ hydatid-specific antigen for immunodiagnosis of hydatidosis," FEMS Immunology and Medical Microbiology, vol. 9, no. 3, pp. 231-236, 1994.

[90] R. M. Matossian, G. N. Awar, H. Radwan, P. S. Craig, and G. A. Meshefedjian, "Immune status during albendazole therapy for hydatidosis," Annals of Tropical Medicine and Parasitology, vol. 86, no. 1, pp. 67-75, 1992.

[91] R. A. Moosa and S. K. Abdel-Hafez, "Serodiagnosis and seroepidemiology of human unilocular hydatidosis in Jordan," Parasitology Research, vol. 80, no. 8, pp. 664-671, 1994.

[92] R. Bonifacino, P. Craig, S. Carter, R. Malgor, and J. Dixon, "Partial characterization of antigens in circulating immune complexes in cystic hydatid patients treated with albendazole," Transactions of the Royal Society of Tropical Medicine and Hygiene, vol. 87, no. 1, pp. 97-102, 1993.

[93] P. Craig, "Immunodiagnosis of Echinococcus granulosus and comparison of techniques for diagnosis of canine echinococcosis," in Compendium on Cystic Echinoccosis in Africa and in Middle Eastern Countries with Special Reference to Morocco, F. L. Andersen, H. Ouhelli, and M. Kachani, Eds., pp. 85-118, Brigham Young University, Provo, Utah, USA, 1997. 
[94] P. M. Schantz, "Circulating antigen and antibody in hydatid disease," The New England Journal of Medicine, vol. 318, no. 22, pp. 1469-1470, 1988.

[95] M. Barbieri, M. A. Severi, M. I. Pirez, J. Battistoni, and A. Nieto, "Use of specific antibody and circulating antigen serum levels in the hydatid immunodiagnosis of asymptomatic population," International Journal for Parasitology, vol. 24, no. 7, pp. 937-942, 1994.

[96] S. Sano, C. L. Smith, and C. R. Cantor, "Immuno-PCR: very sensitive antigen detection by means of specific antibody-DNA conjugates," Science, vol. 258, no. 5079, pp. 120-122, 1992. 


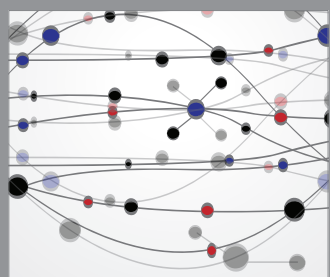

The Scientific World Journal
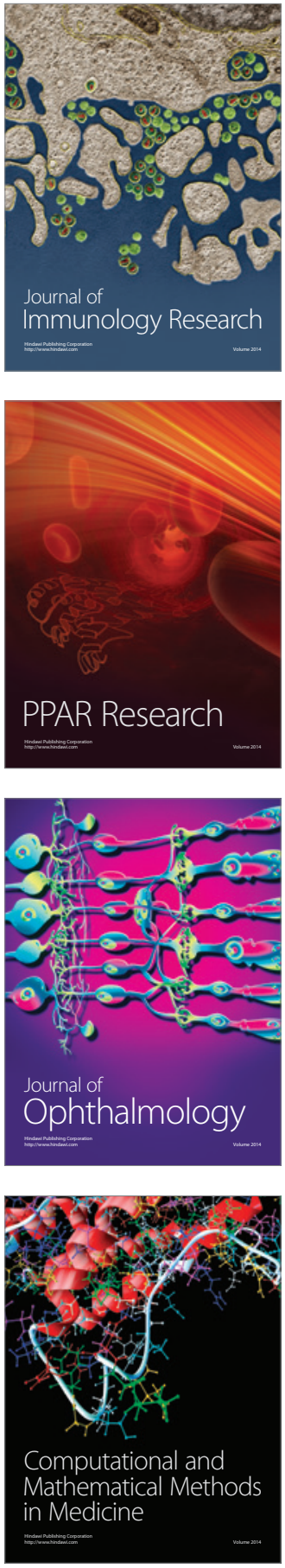

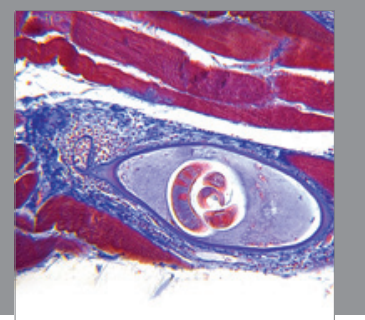

Gastroenterology

Research and Practice
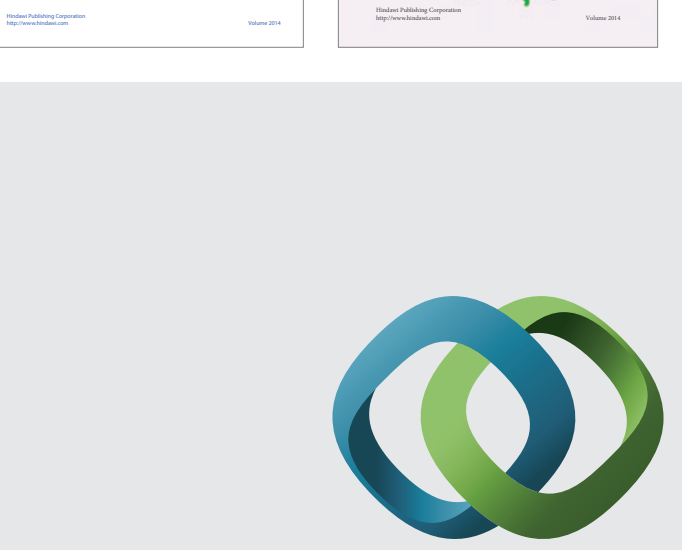

\section{Hindawi}

Submit your manuscripts at

http://www.hindawi.com
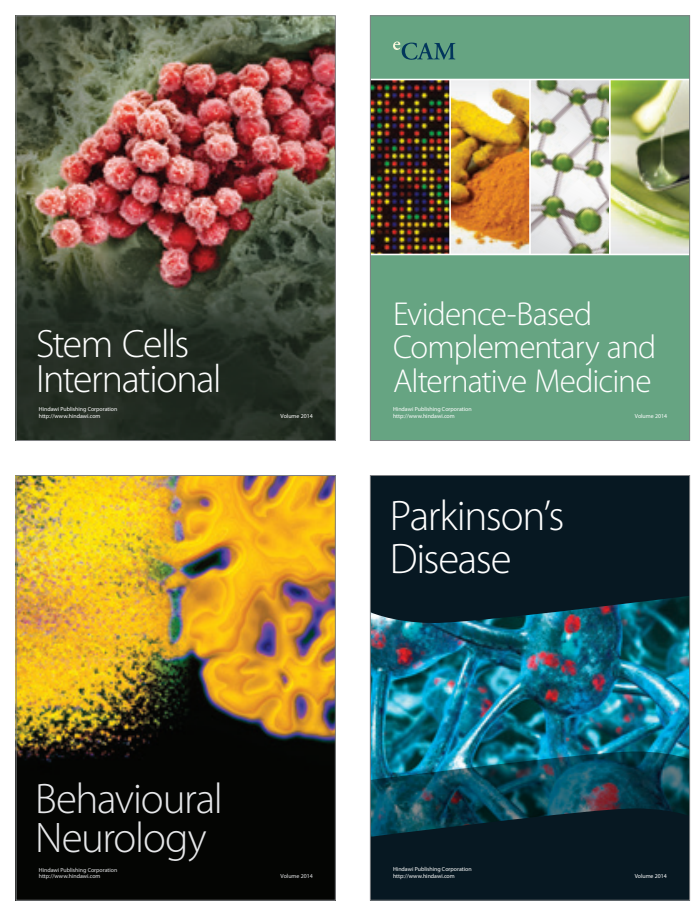

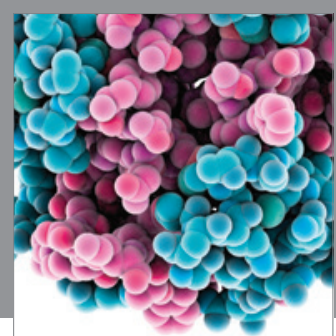

Journal of
Diabetes Research

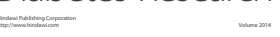

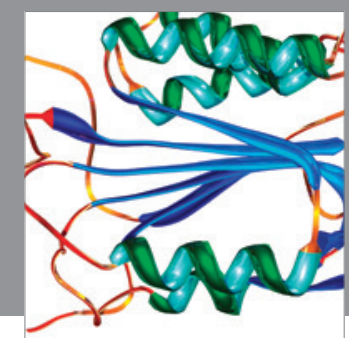

Disease Markers
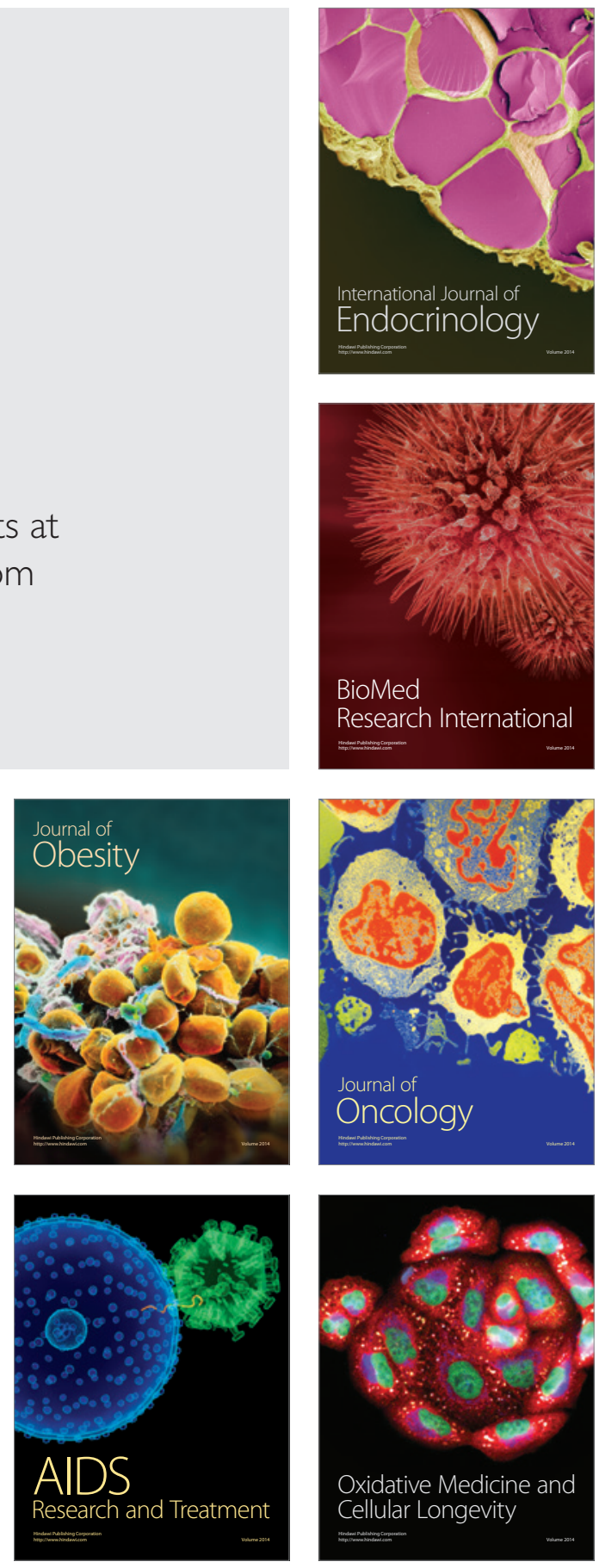